\title{
THE LOCAL DENSITY AND MORPHOLOGY DEPENDENCE OF THE GALAXY LUMINOSITY FUNCTION
}

\author{
Jacek Choloniewski ${ }^{1}$, Miroslaw Panek ${ }^{1,2}$ \\ ${ }^{1}$ Copernicus Astronomical Center, ul. Bartycka 18, \\ 00-716 Warszawa, Poland \\ 2 NASA/Fermilab Astrophysics Group, Chicago, USA
}

We obtained the luminosity function (LF) for samples of galaxies from the CfA North catalogue (Huchra, Davis, Latham and Tonry, 1983). The criteria of selection of samples were the local density (range-more than 2 orders of magnitude) and/or the morphology. No difference in the combined LF for all morphological types is found for subsamples of different density. The LF of elliptical galaxies is found to be less steep at the faint end than the LFs for S and S0 galaxies. E galaxies are on the average brighter than the other morphological types. The LFs measured for earlytype galaxies $(\mathrm{E}+\mathrm{S} 0)$ in high and low density regions show marginal difference- the low density LF has a steeper faint end slope. (Such a difference is not found for $S$ galaxies). If this feature is maintained for larger samples it may indicate that the LF determined at the moment of galaxy formation is only weakly influenced by the phenomena present in dense regions. This is because these phenomena would rather leave the opposite imprint on the LF - the tidal stripping in dense regions would populate them with faint remnants of disrupted, bright, low angular momentum galaxies. Mergers could not reverse this trend because they act mainly on the bright galaxies.

\section{REFERENCES}

Choloniewski, J., Panek, M. 1985, Fermilab preprint 85/179-A

Huchra, J., Davis, M., Latham, D. W., and Tonry, J. 1983, Ap. J. Suppl., 52, 89. 


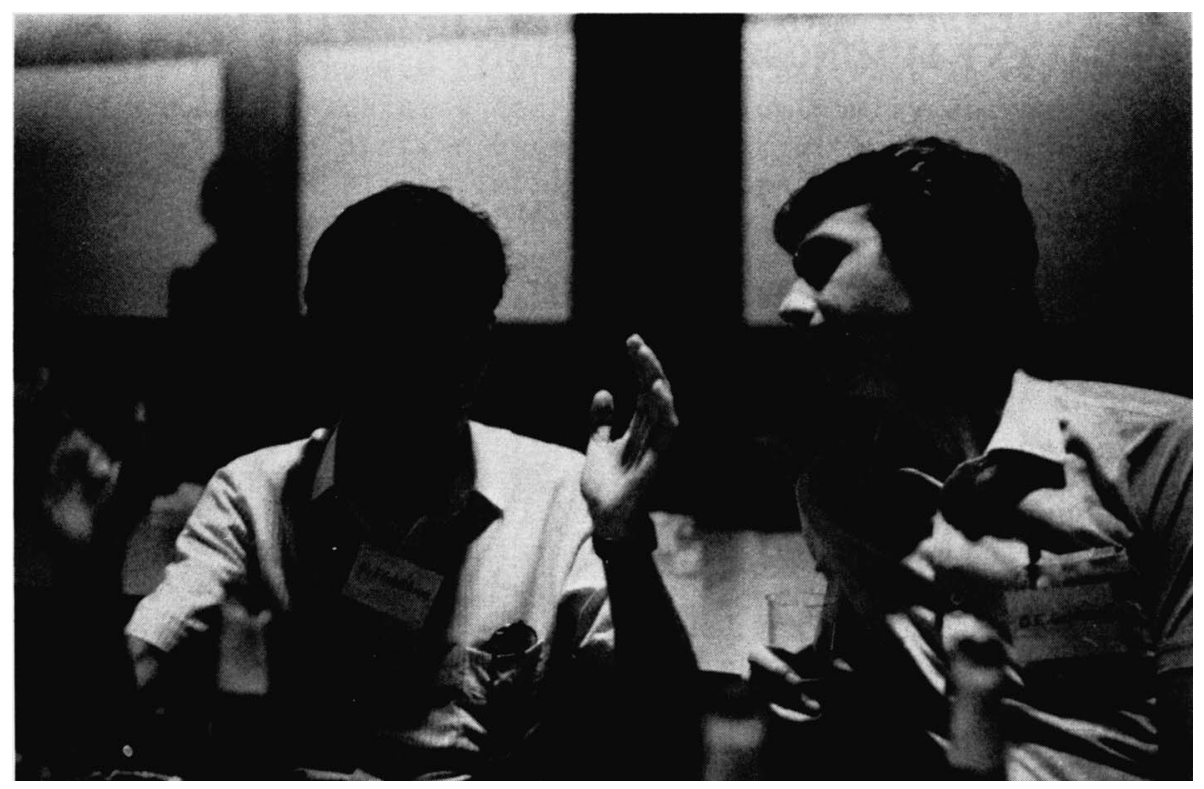

George Efstathiou and Ortwin Gerhard. 